\title{
Anti-Epidermal Growth Factor/Epidermal Growth Factor Receptor Therapeutic Anti-cancer Drugs and the Wound Healing Process
}

\author{
Angel Casacó ${ }^{*}$, Dasha Fuente ${ }^{2}$, Nuris Ledón ${ }^{1}$, Aymara Fernández $^{1}$ and Tania Crombet
}

${ }^{1}$ Centre for Molecular Immunology, Calle 15 esq. 16. Siboney, Playa, Ciudad de la Habana, Cuba. Po Box 16040, Havana 11600, Cuba

${ }^{2}$ Biomodels Unit, National Center for Laboratory Animal Breeding, Finca Tirabeque, Km 2 1/2 Carretera Cacahual, Boyeros, Havana, Postal code 10800, Havana, Cuba

\begin{abstract}
Cutaneous wound healing is a complex process involving blood clotting, inflammation, tissue formation, and tissue remodeling. Many experimental and clinical studies have demonstrated varied, but in most cases beneficial, effects of exogenous growth factors on the healing process. The use of targeted anti-cancer agents is increasing. It is common to utilize a multi-modal treatment approach towards solid tumors, often including surgical resection, and it has become apparent that some targeted agents can impair wound healing or cause increasing risk of perioperative complications. There are limited data regarding the wound healing process of anti-cancer target drugs blocking the EGF/EGFR system. The aim of this paper is to review and to comment the effects of antiEGF/EGFR drugs on the skin wound healing process after programmed or emergency surgical procedures. A review of the current literature, including our own results, was undertaken. We included the monoclonal antibodies cetuximab, panitumumab, nimotuzumab; the small tyrosine kinase molecules erlotinib and gefitinib; and the EGFbased cancer vaccine; CIMAvax and the EGFR-based cancer vaccine; HER-1 vaccine. Apparently, there are no deleterious effects of the anti-EGF/EGFR drugs in the wound healing post-operative process. Taking into account that treatment with anti-EGF/EGFR drugs inhibits tumor cell proliferation, and the lack of deleterious effects of these EGF/EGFR specific inhibitors in the wound healing post-operative process; we suggest that these kinds of drugs could be maintained and their effects tested, with very special surveillance during the post-surgical period.
\end{abstract}

Keywords: Cetuximab; CIMAvax; Erlotinib; Gefitinib; HER-1 vaccine; Nimotuzumab; Panitimumab

\section{Introduction}

Cutaneous wound healing is a complex process involving blood clotting, inflammation, new tissue formation, and finally tissue remodeling. It is well described at the histological level, but the genes that regulate skin repair have only partially been identified. Many experimental and clinical studies have demonstrated varied, but in most cases beneficial, effects of exogenous growth factors on the healing process. However, the roles played by endogenous growth factors have remained largely unclear. Initial approaches at addressing this question focused on the expression analysis of various growth factors, cytokines, and their receptors in different wound models, with first functional data being obtained by applying neutralizing antibodies to wounds. During the past few years, the availability of genetically modified mice has allowed elucidation of the function of various genes in the healing process, and these studies have shed light onto the role of growth factors, cytokines, and their downstream effectors in wound repair [1].

Epidermal growth factor receptor is normally expressed in the epidermis, sebaceous glands, and hair follicular epithelium, where it plays a number of important roles in the maintenance of normal skin health, including control of differentiation, protection against damage induced by ultraviolet radiation, inhibition of inflammation, and acceleration of wound healing. Although the exact mechanism of skin rash mediated by EGFR-targeted monoclonal antibodies is incompletely understood, inhibition of EGFR is believed to cause follicular occlusion and rupture because of premature epithelial differentiation and an increase in the expression of genes that stimulate inflammation, apoptosis, and cell attachment [2].

Table 1 shows the characteristics and dermatologic reactions associated with anti-epidermal growth factor/epidermal growth factor receptor therapeutic anti-cancer drugs.
The epidermal growth factor (EGF) family of mitogens comprises several members, including EGF, transforming growth factor (TGF), heparin-binding EGF (HB-EGF), amphiregulin, epiregulin, betacellulin, neuregulins, the recently discovered epigen, as well as proteins encoded by Vaccinia virus, and other poxviruses $[3,4]$.

The use of targeted anti-cancer agents is increasing. It is common to utilize a multi-modal treatment approach towards solid tumors, often including surgical resection, and it has become apparent that some targeted agents can impair wound healing or cause an increased risk of perioperative complications. A recent article reviews many of the targeted agents used in solid tumor oncology with an emphasis on clinically relevant details [5].

Up to now, evidences on targeted therapy that might cause surgical complications are limited. The greatest amount of data exists for bevacizumab that causes perioperative complications, possibly due to its mechanism of action, neutralizing the ability of all active vascular endothelial growth factor (VEGF) -isoforms to bind to the VEGF receptor (VEGFR) on the surface of endothelial cells [6].

*Corresponding author: Angel Casacó, MD, PhD, Centre for Molecula Immunology, Calle 15 esq. 16. Siboney, Playa, Ciudad de la Habana, Cuba. P O Box 16040, Habana 11600l, Cuba, Tel: (537) 214 3146; Fax: (537) 2720644 E-mail: casaco@cim.sld.cu

Received August 07, 2012; Accepted September 10, 2012; Published Septembe 12, 2012

Citation: Casacó A, Fuente D, Ledón N, Fernández A, Crombet T (2012) AntiEpidermal Growth Factor/Epidermal Growth Factor Receptor Therapeutic Anticancer Drugs and the Wound Healing Process. J Cancer Sci Ther 4: 324-329. doi:10.4172/1948-5956.1000162

Copyright: ( 2012 Casacó A, et al. This is an open-access article distributed under the terms of the Creative Commons Attribution License, which permits unrestricted use, distribution, and reproduction in any medium, provided the original author and source are credited. 


\begin{tabular}{|c|c|c|c|}
\hline Agent & Type & Indication & Most common skin toxicities \\
\hline $\begin{array}{l}\text { Cetuximab } \\
\text { (Erbitux) }\end{array}$ & $\begin{array}{l}\text { Chimeric (mouse/human) } \\
\text { monoclonal antibody }\end{array}$ & Metastatic colorectal cancer and head and neck cancer & \multirow{7}{*}{$\begin{array}{l}\text { Rash (Monomorphous erythematous } \\
\text { maculopapular, follicular, or pustolar lesions which } \\
\text { may be associated with pruritus/tenderness) } \\
\text { Paronychia and fissuring (Painful periungual } \\
\text { granulation-type or friable pyogenic granuloma- } \\
\text { like changes, associated with erythema, swelling, } \\
\text { and fissuring of lateral nailfolds and/or distal finger } \\
\text { tufts) } \\
\text { Hair changes (Alopecia and curlier, finer and more } \\
\text { brittle hair on scalp and extremities; trychomegalia } \\
\text { and curling of eyebrows and hypertrichosis of the } \\
\text { face) }\end{array}$} \\
\hline $\begin{array}{l}\text { Panitumumab } \\
\text { (Vectibix) }\end{array}$ & Fully human monoclonal antibody & $\begin{array}{l}\text { Metastatic colorectal cancer with disease progression } \\
\text { despite prior treatment in patients with non-mutated } \\
\text { (wild-type) KRAS }\end{array}$ & \\
\hline $\begin{array}{l}\text { Nimotuzumab } \\
\text { (CIMAher) }\end{array}$ & Humanized monoclonal antibody & $\begin{array}{l}\text { High grade glioma, esophagus and head and neck } \\
\text { tumor patients }\end{array}$ & \\
\hline $\begin{array}{l}\text { Erlotinib } \\
\text { (Tarceva) }\end{array}$ & $\begin{array}{l}\text { Selective oral inhibitor of EGFR } \\
\text { tyrosine kinase }\end{array}$ & $\begin{array}{l}\text { Locally advanced or metastatic non-small cell lung } \\
\text { cancer after the failure of prior chemotherapy and in } \\
\text { combination with gemcitabine for locally advanced, } \\
\text { unresectable or metastatic pancreatic cancer }\end{array}$ & \\
\hline $\begin{array}{l}\text { Gefitinib } \\
\text { (Iressa) }\end{array}$ & $\begin{array}{l}\text { Selective oral inhibitor of EGFR } \\
\text { tyrosine kinase }\end{array}$ & $\begin{array}{l}\text { Locally advanced or metastatic non-small cell lung } \\
\text { cancer }\end{array}$ & \\
\hline $\begin{array}{l}\text { EGF vaccine } \\
\text { (CIMAvax) }\end{array}$ & $\begin{array}{l}\text { Human recombinant Epidermal } \\
\text { Growth Factor (EGF) chemically } \\
\text { conjugated to a carrier protein from } \\
\text { Neisseria meningitides }\end{array}$ & Advance patients with non-small cell lung cancer & \\
\hline Her-1 Vaccine & $\begin{array}{l}\text { Extracellular domain of human } \\
\text { EGFR/VSSP }\end{array}$ & Hormone refractory prostatic cancer patients & \\
\hline
\end{tabular}

Table 1: Characteristics and dermatologic reactions associated with anti-epidermal growth factor/epidermal growth factor receptor therapeutic anti-cancer drugs.

There are limited data regarding the wound healing process and anti-cancer target drugs blocking the EGF/EGFR system as cetuximab, panitumumab, nimotuzumab, erlotinib, and gefitinib and very little for the EGF-based cancer vaccine CIMAvax and the EGFR-based cancer vaccine HER-1 vaccine.

An analysis of their mechanism of actions suggests, a priory, that there should be an important pharmacovigilence with respect to perioperative complications and increased post-surgical support for patients to aid early detection of postoperative complications until definitive data become available [5].

In this Personal View, we provide an overview of the data, including our own results, for an evidence-based recommendation for the medical treatment with anti-EGF/EGFR drugs and their possible effects on the skin wound healing process of cancer patients undergoing programmed or emergency surgical procedures.

In the present article we are not including the effects of these drugs on cornea and tympanic membrane because diverse and inconsistent kinds of responses have been obtained [7-11].

\section{Agents Targeting EGF/EGFR Pathways}

\section{Antibody-based therapies}

Cetuximab: Cetuximab is a monoclonal antibody targeting EGFR, which is overexpressed in many cancer types. It is licensed by the FDA for its use in patients with metastatic colorectal cancer and locally advanced squamous cell head and neck cancer.

Appropriate management of skin toxicity associated with epidermal growth factor receptor inhibitor therapy is necessary to allow adequate drug administration and to improve quality of life and outcomes.

In a recent study, authors performed a retrospective review of head and neck cancer patients who received salvage neck dissections between 1999 and 2007, at two academic tertiary care centers. Complications from wound healing were compared between radiation and combined therapy groups.

A total of 35 patients received radiation $(n=20)$ or combined radiation and cetuximab therapy $(n=15)$ prior to neck dissection. The treatment groups were similar in regard to demographic and primary tumour-related characteristics. The time between treatment and salvage neck dissection did not differ between the radiation (3.9 months) and combination treatment (3.0 months) groups $(\mathrm{p}=0.15)$. Wound healing complications occurred in $13 \%(2 / 15)$ of the patients treated with radiation and cetuximab and there were no complications in patients who received radiation alone $(\mathrm{p}=0.20)$.

They concluded that cetuximab did not significantly increase the risk of post-surgical wound complications, although a higher absolute number of wound complications was observed in the group treated with cetuximab and radiation therapy, compared with the group treated with radiation alone [12]. These results should be taken into account with caution since patients had surgery six to eight weeks postcetuximab treatment, so these patients have been off cetuximab for two months since the cetuximab's half-life is approximately four days [13].

Panitumumab: Panitumumab is a monoclonal antibody targeting the extracellular domain of the EGFR. It is licensed by the FDA for the treatment of metastatic colorectal cancer. No guidelines have been published regarding the cessation of panitumumab therapy prior to surgery. However, there does not, as yet, appear to be an increase in perioperative complications with this EGFR inhibitor [14].

\section{Small molecule kinase inhibitors}

Erlotinib: Erlotinib is an oral EGFR tyrosine kinase inhibitor indicated for the treatment of patients with locally advanced or metastatic non-small cell lung cancer after the failure of prior chemotherapy and in combination with gemcitabine for locally advanced, unresectable or metastatic pancreatic cancer. There are no reports of surgical complications in patients taking erlotinib [5].

Gefitinib: Gefitinib is a selective inhibitor of EGFR tyrosine kinase and is indicated for the treatment of patients with locally advanced or metastatic non-small cell lung cancer. It also represents a potential therapeutic target for head and neck squamous cell carcinoma (HNSCC). In vitro and in vivo studies have demonstrated that the combination of INF-alpha and gefitinib might have a cooperative antitumor effect of HNSCC-derived cell lines [15-17].

A case series was published describing four patients who underwent different types of surgery (emergency laparotomy, internal fixation for bone metastases and incision, and drainage of labial abscess) concurrently with gefitinib with no adverse effect on wound healing [18]. 


\section{The Center of Molecular Immunology's Strategy}

The Center of Molecular Immunology (CIM) in Havana, Cuba, has developed its own strategy, blocking the EGFR pathway with monoclonal antibodies (i.e., nimotuzumab) and with cancer vaccines (EGF-vaccine and HER-1 vaccine). The monoclonal antibody nimotuzumab and the EGF-vaccine have been registered in Cuba and other Countries and HER-1 vaccine is in a Phase I Clinical Trial for treating cancer diseases from epithelial origin.

The fact that our registered drugs have demonstrated evidences of clinical benefit with absence of serious toxicities, including during concomitant chemo-radiotherapy, supports the possibility of a long lasting administration period in patients with cancer from epithelial origin.

Several studies are ongoing for demonstrating that our combination of targeting techniques with conventional current treatment protocols may improve the treatment outcome and disease control, without exacerbating the treatment related toxicities.

\section{Nimotuzumab}

Nimotuzumab is an anti-EGFR monoclonal antibody registered in 26 markets and in Cuba by the Cuban regulatory Agency, CECMED, for treating high grade glioma, head and neck and esophageal cancer patients.

It was originally generated as a mouse IgG2a antibody and humanized by grafting its complementarity-determining regions (CDRs) to a human IgG1 gene.

In several trials, it has shown activity and been well tolerated when used together with radiation [19]. Preclinical data have suggested that nimotuzumab may enhance the anti-tumor activity of ionizing radiation [20,21]. Clinical experience has demonstrated good tolerability of nimotuzumab at $50-800 \mathrm{mg}$ doses when given as a single agent, [22] or in combination with radiation to head and neck cancer patients $[23,24]$.

The low toxicity profile shown by nimotuzumab has allowed its use in prolonged treatments, lasting several months, and even years in many cases. About two thirds of the 600 patients that have been treated in Cuba since 2002 have received more than six doses of the antibody (200 $\mathrm{mg}$ for adult, $150 \mathrm{mg} / \mathrm{m}^{2}$ for children), including about 50 patients that have been treated with more than 30 , bi-weekly doses during more than one year. In particular, two children with brain stem glioma tumors have received more than 100 doses of nimotuzumab, continuously for more than three years, without showing adverse effects. It is worth noting that the frequency of adverse events (limited to grade 1 or 2) observed in these patients did not increase with drug exposure $[25,26]$.

From this clinical experience we have obtained important evidences on the impact of chronic treatment in disease stabilization and increase of overall survival in advanced cancer patients. In more than 20,000 patients treated world-wide with nimotuzumab, there are no reports of adverse events associated with surgical complications [27-29].

\section{Cancer vaccines}

Therapeutic vaccines continue to be one of the most active fields in cancer research. However, despite clear evidence of antitumor effect in laboratory animals, and despite the ability of current vaccine candidates to elicit tumor specific antibodies and T-cells in humans, objective responses in the clinical trials are rare. Actually, only sipuleucel-T (Provenge), a prostatic acid phosphatase-granulocyte/ macrophage- colony-stimulating factor (PAP-GM-CSF) fusion protein-loaded autologous blood cell vaccine, has been approved by the US Food and Drug Administration (FDA) in April 2010 for the treatment of asymptomatic or minimally symptomatic metastatic castration-resistant prostate cancer patients [28].

The role of therapeutic vaccines in advanced cancer patients, if any, would be to decrease the rate of disease progression and to increase survival and quality of life. Due to the redundant regulatory loops contracting the immune response to antigens that cannot be eliminated, such a role would require chronic vaccination, which is at first sight at odds with the classic experience of vaccinology.

\section{EGF-vaccine}

During the last decade, our team has developed a therapeutic vaccine for advanced lung cancer, which consists of human recombinant Epidermal Growth Factor (EGF) chemically conjugated to a carrier protein from Neisseria meningitides. Several clinical trials have been carried out, showing increase in anti-EGF antibody titers, decrease in plasma EGF concentration and survival advantage in vaccinated patients bearing advanced non-small cell lung cancer (NSCLC) [29]. This EGF-based cancer vaccine (CIMAvax) has been approved by the Cuban regulatory Agency, CECMED, in 2008 for treating advanced patients with NSCLC.

In a recent article, authors reported that 58 patients were vaccinated monthly for more than one or two years, and this long term vaccination was feasible and safe, and there was no evidence of wound healing or any cumulative toxicity [30].

In a non-clinical study, authors from the same group investigated the possible role of the EGF-vaccine in the croton-oil-induced ear edema and in the wound healing experimental animal models.

Mice were immunized with an EGF-vaccine by intramuscular injections and serum titers against EGF were measured through ELISA techniques. Control animals received saline.

Immunized mice produced antibodies against EGF while no antibody titers could be measured in control animals. Croton oil applied to the inner ear surface of EGF-vaccine treated mice caused a $61.3 \%$ lower ear punch weight and a $60.2 \%$ lower myeloperoxidase activity than control mice. In the EGF-vaccine treated animals, planimetry measurements and histological analysis did not lead to significant impairment in tissue repair.

The authors concluded that EGF-vaccination in mice decreased the normal croton-oil-induced inflammation response, without apparent impairment in tissue healing [31].

\section{HER-1 vaccine}

HER-1 vaccine is a cancer vaccine which is in its very beginning clinical steps in Cuba. In non-clinical pharmacodynamics studies Ramírez and colleagues [32] immunized mice with the extracellular domain of murine EGFR (mEGFR-ECD) in adjuvants with the objective of circumvent tolerance to self EGFR by inducing an immune response with consequent antitumor effect. The study demonstrated that despite mEGFR expression in thymus, strong DTH response was induced by inoculation of mice with the mEGFR-ECD. This self-immunization, using both Freund's Complete Adjuvant (FCA) and very small sized 
proteoliposomes from Neisseria meningitidis (VSSP), promoted highly specific IgG titers, predominantly IgG2a and IgG2b. Sera from mice immunized with mEGFR-ECD/VSSP not only recognized EGFR1 tumor cell lines by FACS, but also inhibited the in vitro growth, even in the absence of complement. Noteworthy, vaccination of mice with mEGFR-ECD/VSSP stimulated a potent anti-metastatic effect in the EGFR Lewis lung carcinoma model; while reproduction associated side effects were absent. Curiously, mice immunized with the human EGFR-ECD (Her1-ECD) in VSSP though induced highly specific IgG antibodies with strong in vitro cytotoxic effect over EGFR human cell lines, showed low cross-reactivity with the mEGFR-ECD.

Fuentes et al. [33], explore the possible role of mEGFR-ECD vaccine in the croton-oil-induced ear edema and the wound healing in experimental animal models. Mice were immunized four times biweekly with $50 \mu \mathrm{g}$ of mEGFR-ECD/VSSP/Montanide or with PBS/ VSSP/Montanide by intramuscular way. Mice treated with mEGFR$\mathrm{ECD} / \mathrm{VSSP} / \mathrm{Montanide}$ had a similar inflammatory response compared to control ones in the croton oil inflammation model. No differences in healing speed were found for the skin wounds in the mice vaccinated with mEGFR-ECD, with respect to the control animals. Planimetry measurements, histological and morphometrical analysis did not lead to significant impairment in tissue repair. These data suggest that application of Her1-ECD/VSSP vaccine as a therapeutic approach in cancer patients would not elicit a poor healing process after surgery or other invasive procedures.

An immune-active dose-escalation Phase I Clinical Trial in hormone refractory prostatic cancer patients treated with the ECD of the human EGFR is on-going in Cuba, and especial emphasis is performing in detecting any skin or wound healing adverse event.

A schematic representation of the anti-EGF/EGFR therapeutic anti-cancer drugs and their mechanisms of actions are shown in figure 1 .

\section{Reflections and Conclusions}

Several reports have shown that post-operative leakage may have a negative impact on long-term survival in patients with malignancies [34-39].

Evidences suggest that recurrence of resected head and neck squamous cell carcinomas (HNSCCs) is due to the outgrowth of unrecognized residual tumor cells as well as to the premalignant and/ or precursor-field epithelial cells. Licitra et al. [40] studied the impact of processes triggered by HNSCC surgery in stimulating both residual tumor cells, demonstrated to overexpress epidermal growth factor receptor (EGFR), and premalignant cells surrounding the resected lesion.

They showed that primary tumors have intermediate/high EGFR expression (91\%), EGFR phosphorylation and EGFRpositive FISH (35\%). Normal, metaplastic and dysplastic epithelium surrounding the resected tumor displayed EGFR overexpression. EGFR activation and gene amplification were observed in normal and dysplastic epithelium, respectively. Each tested wound healing drainage induced cells to proliferate and the proliferation was significantly higher in relapsed compared with non-relapsed HNSCC patients. Anti-EGFR treatments inhibited the drainage-induced proliferation, with the highest inhibitory efficiency by cetuximab on A431 cells, while CAL27 cell growth was more efficiently inhibited by tyrosine kinase inhibitors.

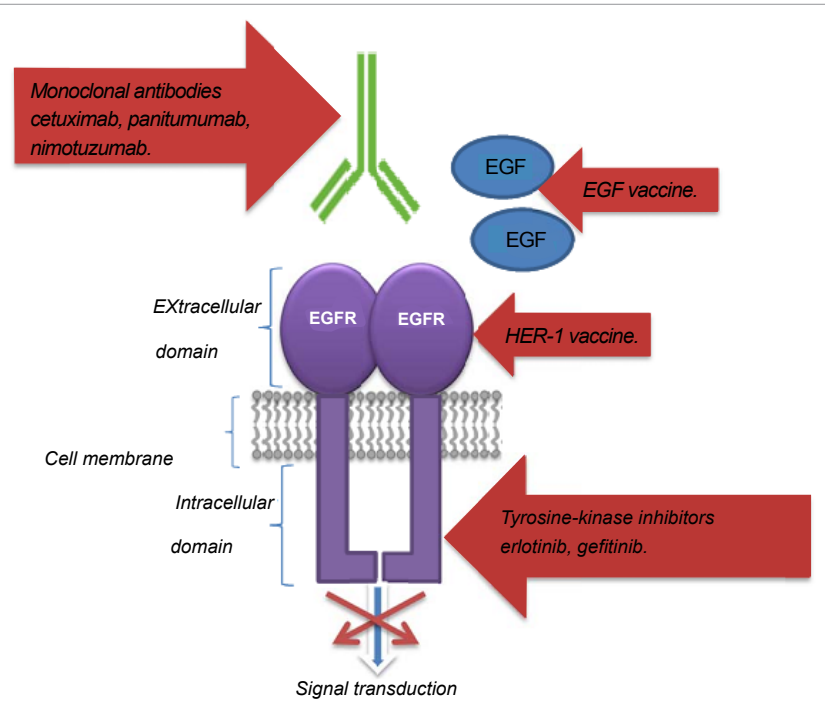

Figure 1: Monoclonal antibodies cetuximab, panitumumab, and nimotuzumab; tyrosine-kinase inhibitor serlotinib and gefitinib; and EGF and HER-1 cancer vaccines are target treatments that produce inhibition of signal transduction.

They concluded that surgery could favor the proliferation of cells showing EGFR overexpression/activation/amplification such as residual tumor cells and/or precursor-field epithelial cells already present after surgery and that treatment with anti- EGFR reagents inhibits wound-induced stimulation, according to the EGFR family status. Similar results have been obtained in breast cancer with the administration of preoperative chemotherapy and trastuzumab [41].

Brusevold et al. [42] showed, using a scratch wound assay in vitro, that EGF and hepatocyte growth factor (HGF) induce cell migration in human oral squamous carcinoma cell lines and that addition of EGFRspecific inhibitors cetuximab or gefitinib abolishes cell migration elicited by EGF.

In a more recently study, Sano et al. [43] used a nice combination of preclinical animal models. In the first study an athymic nude BALB/c mouse model of human microscopic residual oral squamous cell carcinoma (OSCC) were used, mice were treated with cetuximab with the objective of measuring tumor growth, survival, and cervical lymph node metastases.

In the second study, a BALB/c normal mouse model of wound healing was established to assess the effect of an EGFR antibody on the wound healing process, in this experiment authors used the ratantimouse EGFR antibody ME-1.

They hypothesized that EGF and transforming growth factor (TGF)-a are present in the wounds of patients who have undergone resection of head and neck cancers and could be stimulating the early repopulation of residual tumor cells and that inhibition of EGFR signaling in this setting could inhibit this tumor regrowth and thereby improve treatment outcomes.

They demonstrated that cetuximab inhibited tumor progression in the in vivo model of microscopic residual disease and that the use of a rat-anti mouse EGFR antibody does not significantly delay the process of wound healing in a mouse wound healing model. They demonstrated in a preclinical model that intervention in the EGFR pathway with 
cetuximab treatment soon after surgery could inhibit the repopulation of OSCC cells without impairing wound healing [43].

On the other hand, it has been recently shown, using a wound healing assay, that EGFR is also involved in radiation induced migration of HNSCC cells and therefore EGFR might be a target for the treatment of tumors to improve the efficacy of radiation [44].

Considering the above discussed elements, seems that treatment with anti-EGF/EGFR drugs, apparently, does not elicit a deleterious effect in the wound healing process, inhibits wound-induced tumor cell proliferation, and radiation induced cell migration; we suggest that these kinds of drugs would still be useful in the wound healing postoperative process. The treatment might be maintained and their effects tested, with very special surveillance, at least in HNSCC patients during the post-surgical period. Obviously, further researches are needed for better understanding of these pathophysiological processing HNSCCs and in other EGFR over-expressing cancers from epithelial origin.

\section{References}

1. Werner S, Grose R (2003) Regulation of wound healing by growth factors and cytokines. Physiol Rev 83: 835-870.

2. Melosky B, Burkes R, Rayson D, Alcindor T, Shear N, et al. (2009) Management of skin rash during EGFR-targeted monoclonal antibody treatment for gastrointestinal malignancies: Canadian recommendations. Curr Oncol 16: 1626.

3. Strachan L, Murison JG, Prestidge RL, Sleeman MA, Watson JD, et al. (2001) Cloning and biological activity of epigen, a novel member of the epidermal growth factor superfamily. J Biol Chem 276: 18265-18271.

4. Yarden Y (2001) The EGFR family and its ligands in human cancer. signalling mechanisms and therapeutic opportunities. Eur J Cancer 37: S3-S8.

5. Mellor JD, Cassumbhoy M, Jefford M (2011) Clinical guidance on the perioperative use of targeted agents in solid tumor oncology. Asia Pac J Clin Oncol 7: 106-113.

6. Hompes D, Ruers T (2011) Review: incidence and clinical significance of Bevacizumab-related non-surgical and surgical serious adverse events in metastatic colorectal cancer. Eur J Surg Oncol 37: 737-746.

7. Nakamura Y, Sotozono C, Kinoshita S (2001) The epidermal growth factor receptor (EGFR): role in corneal wound healing and homeostasis. Exp Eye Res 72: 511-517.

8. Foerster CG, Cursiefen C, Kruse FE (2008) Persisting corneal erosion unde cetuximab (Erbitux) treatment (epidermal growth factor receptor antibody). Cornea 27: 612-614.

9. Kaftan H, Reuther L, Miehe B, Hosemann W, Herzog M (2008) Delay of tympanic membrane wound healing in rats with topical application of a tyrosine kinase inhibitor. Wound Repair Regen 16: 364-369.

10. Lee SM, Buchler T, Joseph T, Lai C (2008) Bilateral eardrum perforation after long-term treatment with erlotinib. J Clin Oncol 26: 2582-2584.

11. Márquez EB, De Ortueta D, Royo SB, Martínez-Carpio PA (2011) Epiderma growth factor receptor in corneal damage: update and new insights from recent reports. Cutan Ocul Toxicol 30: 7-14.

12. Dean NR, Sweeny L, Harari PM, Bonner JA, Jones V, et al. (2011) Wound healing following combined radiation and cetuximab therapy in head and neck cancer patients. J Wound Care 20: 166-170.

13. Fracasso PM, Burris H 3rd, Arquette MA, Govindan R, Gao F, et al. (2007) A phase 1 escalating single-dose and weekly fixed-dose study of cetuximab: pharmacokinetic and pharmacodynamic rationale for dosing. Clin Cancer Res 13: $986-993$.

14. Parikh AA, Ellis LM (2008) Targeted therapies and surgical issues in gastrointestinal cancers. Target Oncol 3: 119-125.

15. Caraglia M, Leardi A, Corradino S, Ciardiello F, Budillon A, et al. (1995) alpha-
Interferon potentiates epidermal growth factor receptor-mediated effects on human epidermoid carcinoma KB cells. Int J Cancer 61: 342-347.

16. Caraglia M, Vitale G, Marra M, Budillon A, Tagliaferri P, et al. (2004) Alphainterferon and its effects on signalling pathways within cells. Curr Protein Pept Sci 5: 475-485

17. Bruzzese F, Di Gennaro E, Avallone A, Pepe S, Arra C, et al. (2006) Synergistic antitumor activity of epidermal growth factor receptor tyrosine kinase inhibitor gefitinib and IFN-alpha in head and neck cancer cells in vitro and in vivo. Clin Cancer Res12: 617-625.

18. Govindan R, Behnken D, Read W, McLeod H (2003) Wound healing is no impaired by the epidermal growth factor receptor-tyrosine kinase inhibitor gefitinib. Ann Oncol 14: 1330-1331.

19. Crombet T, Osorio M, Cruz T, Roca C, del Castillo R, et al. (2004) Use of the humanized anti-epidermal growth factor receptor monoclonal antibody h-R3 in combination with radiotherapy in the treatment of locally advanced head and neck cancer patients. J Clin Oncol 22: 1646-1654.

20. Akashi Y, Okamoto I, Iwasa T, Yoshida T, Suzuki M, et al. (2008) Enhancemen of the antitumor activity of ionising radiation by nimotuzumab, a humanised monoclonal antibody to the epidermal growth factor receptor, in non-small cell lung cancer cell lines of differing epidermal growth factor receptor status. $\mathrm{Br} J$ Cancer 98: 749-755.

21. González JE, Barquinero JF, Lee M, García O, Casaco A (2012) Radiosensitization induced by the anti-epidermal growth factor recepto monoclonal antibodies cetuximab and nimotuzumab in A431 cells. Cancer Biol Ther 13: 71-76.

22. You B, Brade A, Magalhaes JM, Siu LL, Oza A, et al. (2011) A dose-escalation phase I trial of nimotuzumab, an antibody against the epidermal growth facto receptor, in patients with advanced solid malignancies. Invest New Drugs 29: 996-1003.

23. Rojo F, Gracias E, Villena N, Cruz T, Corominas JM, et al. (2010) Pharmacodynamic trial of nimotuzumab in unresectable squamous cell carcinoma of the head and neck: a SENDO Foundation study. Clin Cancer Res 16: $2474-2482$

24. Rodríguez MO, Rivero TC, del Castillo Bahi R, Muchuli CR, Bilbao MA, et al. (2010) Nimotuzumab plus radiotherapy for unresectable squamous-cel carcinoma of the head and neck. Cancer Biol Ther 9: 343-349.

25. Allan DG (2005) Nimotuzumab: evidence of clinical benefit without rash Oncologist 10: 760-761.

26. Saurez G, Cabanas R, Zaldívar M, Garnier T, Iglesias B, et al. (2009) Clinica experience with nimotuzumab in cuban pediatric patients with brain tumors, 2005 to 2007. MEDICC Rev 11: 27-33.

27. Perez R, Moreno E, Garrido G, Crombet T (2011) EGFR-Targeting as a Biological Therapy: Understanding Nimotuzumab's Clinical Effects. Cancers 3 : 2014-2031.

28. Garcia JA, Dreicer R (2011) Immunotherapy in castration-resistant prostate cancer: integrating sipuleucel-T into our current treatment paradigm. Oncology (Williston Park) 25: 242-249.

29. Rodriguez G, Gonzalez G, Crombet T, Lage A (2011) Therapeutic vaccination with an EGF-based vaccine in lung cancer: a step in the transition to a chronic disease. Expert Rev Respir Med 5: 337-342.

30. Gonzalez G, Crombet T, Lage A (2011) Chronic vaccination with a therapeutic EGF-based cancer vaccine: a review of patients receiving long lasting treatment. Curr Cancer Drug Targets 11: 103-110.

31. Casacó A, Díaz Y, Ledón N, Merino N, Vadés O, et al. (2004) Effect of an EGF-cancer vaccine on wound healing and inflammation models. J Surg Res 122: $130-134$.

32. Ramírez BS, Pestana ES, Hidalgo GG, García TH, Rodríguez RP, et al. (2006) Active antimetastatic immunotherapy in Lewis lung carcinoma with self EGFR extracellular domain protein in VSSP adjuvant. Int J Cancer 119: 2190-2199.

33. Fuentes D, Chacón L, Casacó A, Ledón N, Fernández N, et al. (2012) Effects of an epidermal growth factor receptor-based cancer vaccine in the wound healing and inflammation processes in murine experimental models. Int Wound J. 
Citation: Casacó A, Fuente D, Ledón N, Fernández A, Crombet T (2012) Anti-Epidermal Growth Factor/Epidermal Growth Factor Receptor Therapeutic Anti-cancer Drugs and the Wound Healing Process. J Cancer Sci Ther 4: 324-329. doi:10.4172/1948-5956.1000162

34. Bell SW, Walker KG, Rickard MJ, Sinclair G, Dent OF, et al. (2003) Anastomotic leakage after curative anterior resection results in a higher prevalence of local recurrence. Br J Surg 90: 1261-1266.

35. Junemann-Ramirez M, Awan MY, Khan ZM, Rahamim JS (2005) Anastomotic leakage post-esophagogastrectomy for esophageal carcinoma: retrospective analysis of predictive factors, management and influence on longterm survival in a high volume centre. Eur J Cardiothorac Surg 27: 3-7.

36. McArdle CS, McMillan DC, Hole DJ (2005) Impact of anastomotic leakage on long-term survival of patients undergoing curative resection for colorectal cancer. Br J Surg 92: 1150-1154.

37. Law WL, Choi HK, Lee YM, Ho JW, Seto CL (2007) Anastomotic leakage is associated with poor long-term outcome in patients after curative colorectal resection for malignancy. J Gastrointest Surg 11: 8-15.

38. Lee WS, Yun SH, Roh YN, Yun HR, Lee WY, et al. (2008) Risk factors and clinical outcome for anastomotic leakage after total mesorectal excision for rectal cancer. World J Surg 32: 1124-1129.

39. Yoo HM, Lee HH, Shim JH, Jeon HM, Park CH, et al. (2011) Negative impact of leakage on survival of patients undergoing curative resection for advanced gastric cancer. J Surg Oncol 104: 734-740.
40. Licitra L, Perrone F, Tamborini E, Bertola L, Ghirelli C, et al. (2011) Role of EGFR family receptors in proliferation of squamous carcinoma cells induced by wound healing fluids of head and neck cancer patients. Ann Oncol 22: 18861893.

41. Tagliabue E, Agresti R, Carcangiu ML, Ghirelli C, Morelli D, et al. (2003) Role of HER2 in wound-induced breast carcinoma proliferation. Lancet 362: 527-533.

42. Brusevold IJ, Aasrum M, Bryne M, Christoffersen T (2012) Migration induced by epidermal and hepatocyte growth factors in oral squamous carcinoma cells in vitro: role of MEK/ERK, p38 and PI-3 kinase/Akt. J Oral Pathol Med 41: 547-558.

43. Sano D, Gule MK, Rosenthal DI, Bell D, Yates J, et al. (2012) Early postoperative epidermal growth factor receptor inhibition: Safety and effectiveness in inhibiting microscopic residual of oral squamous cell carcinoma in vivo. Head Neck.

44. Pickhard AC, Margraf J, Knopf A, Stark T, Piontek G, et al. (2011) Inhibition of radiation induced migration of human head and neck squamous cell carcinoma cells by blocking of EGF receptor pathways. BMC Cancer 11: 388. 